\title{
LA LOI DE KIRCHHOFF
}

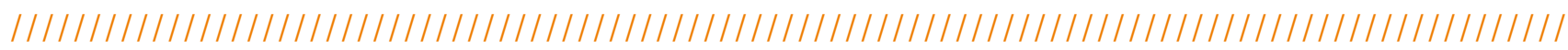

\section{Jean-Jacques GREFFET}

Université Paris-Saclay, Institut d'Optique Graduate School, CNRS, Laboratoire Charles Fabry, 91127 Palaiseau, France jean-jacques.greffet@institutoptique.fr

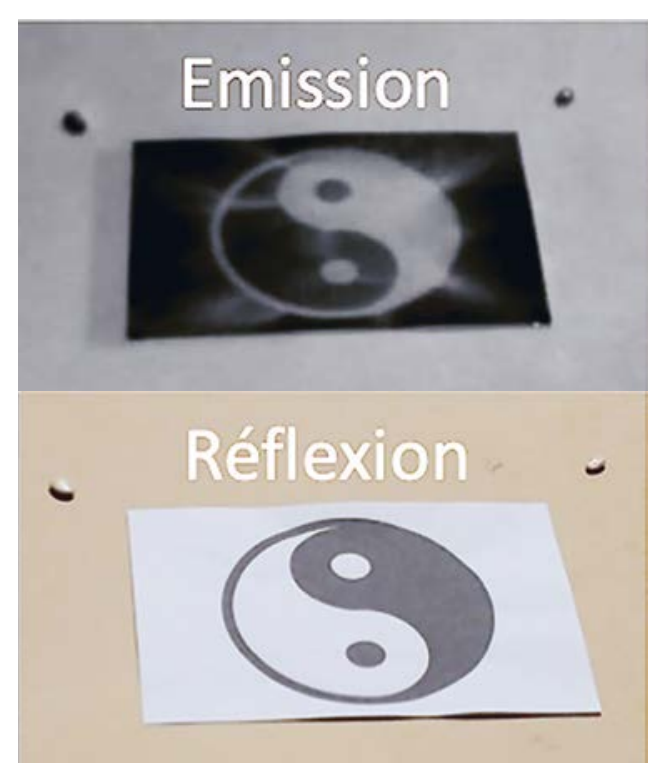

(9 incandescence est souvent associée à l'éclairage par filament incandescent. Avant d'être une technologie d'éclairage, le rayonnement du corps noir a longtemps été une énigme scientifique. L'étude thermodynamique du rayonnement a conduit Planck à introduire sa célèbre constante $h$. Aujourd'hui, les mesures du rayonnement émis par les étoiles ou du fond cosmologique continuent à jouer un rôle essentiel en astrophysique et en cosmologie. À de plus petites échelles de taille, le rayonnement thermique nous a réservé de nombreuses surprises avec l'avènement des nanosciences. Nous avons appris à le rendre spectralement étroit, directionnel et à augmenter les flux d'énergie bien au-delà de celui d'un corps noir.

L'incandescence est le phénomène d'émission de rayonnement par des corps chauffés. L'aptitude d'un corps à émettre de la lumière a été reliée à son absorptivité par Kirchhoff. Ce texte retrace l'évolution de cette loi depuis son introduction en $\mathbf{1 8 6 0}$ dans le cadre de l'optique géométrique, les développements qui ont suivi lorsqu'elle a pu être revisitée dans un cadre d'électromagnétisme stochastique, aux échelles nanométriques et enfin dans des situations hors équilibre telles que des semiconducteurs pompés électriquement.

\section{https://doi.org/10.1051/photon/202010546}

Article publié en accès libre sous les conditions définies par la licence Creative Commons Attribution License CC-BY (https://creativecommons.org/licenses/by/4.0), qui autorise sans restrictions l'utilisation, la diffusion, et la reproduction sur quelque support que ce soit, sous réserve de citation correcte de la publication originale.

Dans tous ces exemples, la loi de Kirchhoff est le concept de base utilisé pour décrire l'émission. Le but de cet article est de présenter un bref aperçu historique de la formulation de la loi de Kirchhoff qui date de 1860 puis de montrer comment cette loi a été comprise, réinterprétée et généralisée plusieurs fois.

\section{LA LOI DE KIRCHHOFF : UN APERÇU HISTORIQUE}

En 1860, G. Kirchhoff a publié dans Poggendorf Annalen, un article intitulé «Sur la relation entre la puissance radiante et la puissance absorbée de différents corps pour la lumière et la chaleur ». Une traduction anglaise est disponible [1]. Dès le début de son article, Kirchhoff rappelle qu'à l'équilibre, la puissance totale émise doit être égale à la puissance totale absorbée du fait de la conservation de l'énergie. La question qu'il traite est celle de l'égalité entre l'absorptivité et l'émissivité pour une fréquence, une polarisation et un angle d'incidence donnés, ce que nous pourrions appeler aujourd'hui un bilan détaillé.

Ce questionnement est notamment motivé par l'observation du doublet du sodium brillant en émission et sombre en transmission pour les deux fréquences du doublet. Dans son raisonnement, il est amené à introduire le désormais célèbre «corps noir » qui est défini comme un corps ayant une surface parfaitement absorbante à toute longueur d'onde. Il utilise des bilans d'énergie sur des systèmes comportant un corps à l'intérieur d'une enceinte qui comporte de petites ouvertures munies de polariseurs et de filtres. L'analyse des flux échangés, qui est menée dans 
le cadre de l'optique géométrique, le conduit à utiliser la réciprocité de Helmholtz. La formulation de sa loi en notations modernes est :

$$
\varepsilon(v, \mathbf{u}, 1)=a(v,-\mathbf{u}, 1)
$$

où l'absorptivité $a(v,-u, l)$ est la fraction absorbée de la puissance incidente de fréquence $v$, se propageant dans la direction -u, et de polarisation 1 et $\varepsilon(v, \mathbf{u}, 1)$ est l'émissivité du corps de température $\mathrm{T}$ définie $\operatorname{par} \mathrm{L}^{\mathrm{e}}(v, \mathbf{u}, 1, \mathrm{~T})=\varepsilon(v, \mathbf{u}, 1) \mathrm{L}^{\circ}(v, \mathrm{~T})$, où $\mathrm{L}^{\mathrm{e}}(v, \mathbf{u}, 1, \mathrm{~T})$ est la luminance émise et $\mathrm{L}^{\circ}(v, \mathrm{~T})$ est la luminance du rayonnement à l'équilibre thermodynamique à T. On voit ainsi qu'un corps noir $(a(v, \mathbf{u}, 1)=1)$ émet une luminance égale à la luminance du rayonnement d'équilibre ce qui explique que ces deux notions soient souvent interchangeables dans l'usage courant. Cette loi est illustrée par l'image du Yin et du Yang effectuée avec de la peinture noire sur du papier blanc (voir la figure en début d'article). Les zones brillantes observées en émission infrarouge avec une caméra thermique fonctionnant dans la bande 8-12 $\mu \mathrm{m}$ sont celles qui correspondent à la peinture noire qui absorbe fortement dans cette bande. Notons que lorsque Kirchhoff établit cette loi en 1860, Planck n'avait pas encore établi la luminance du rayonnement à l'équilibre. Rappelons également que le traité d'électricité et de magnétisme de Maxwell date de 1873.

L'approche adoptée par Kirchhoff repose sur des bilans d'énergie et ne donne aucun indice sur les mécanismes physiques responsables de l'émission et de l'absorption. Dans le postscriptum qui apparaît dans la traduction anglaise de l'article, Kirchhoff rapporte avoir eu une correspondance avec Stokes qui lui a suggéré une explication «mécanique » à la relation existant entre absorption et émission. Nous dirions aujourd'hui que l'émission spontanée et l'absorption sont toutes deux décrites au moyen du même élément de matrice décrivant la transition entre deux niveaux d'énergie.
Il y a donc d'un côté, le phénomène d'émission qui est de nature thermodynamique ou statistique, de l'autre un phénomène d'absorption par un corps éclairé par une onde cohérente. La loi de Kirchhoff établit un pont entre la thermodynamique et l'optique cohérente, rendu nécessaire par la conservation de l'énergie mais sans nous en fournir le mécanisme physique sous-jacent.

\section{COMPRENDRE LA LOI DE KIRCHHOFF EN DEUX SCHÉMAS}
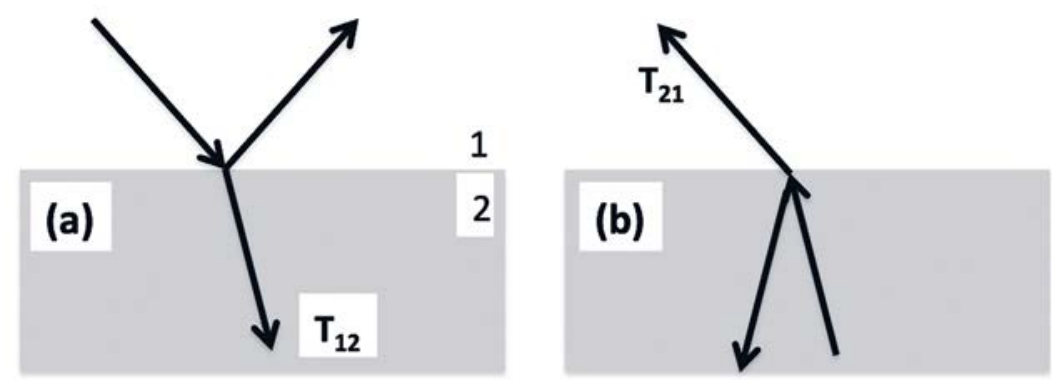

On considère une interface séparant du vide (milieu 1) d'un milieu absorbant semi infini (milieu 2). Sur la figure (a), un rayon est partiellement transmis et partiellement réfléchi. L'énergie transmise sera absorbée dans le milieu 2 qui est semi infini. Il en résulte qu'il y a identité entre l'absorptivité du corps $\alpha$ et le facteur de transmission $\mathrm{T}_{12}$ en intensité du milieu 1 vers le milieu 2. Sur la figure (b), on représente le milieu 2 qui est à l'équilibre thermodynamique local à la température $T$ de sorte que la matière et le rayonnement sont en équilibre. Il existe ainsi dans ce milieu un rayonnement qui peut se propager vers l'interface quand bien même ce serait sur de faibles distances si le milieu est très absorbant. Lorsqu'un rayon atteint l'interface (voir Fig.b), il est partiellement transmis et partiellement réfléchi. La partie transmise est ainsi proportionnelle au facteur de transmission $T_{21}$ et à la luminance du corps noir à la température du corps. Le facteur de transmission $T_{21}$ peut donc $s$ 'identifier à l'émissivité. Du fait de la réciprocité de Helmholtz, $T_{12}=T_{21}$ ce qui entraine $\alpha=\varepsilon$. On voit ainsi pourquoi la réciprocité joue un rôle essentiel dans la loi de Kirchhoff: l'absorptivité et l'émissivité traduisent toutes deux la transmission entre l'intérieur et l'extérieur du corps émetteur.

Avec cette image, l'émissivité d'un corps est simplement le facteur de transmission de l'interface. Il est donc en principe possible de faire en sorte que ce facteur de transmission soit sélectif en fréquence ou en direction de sorte que l'on peut en principe contrôler la directivité et le spectre du rayonnement émis. En pratique cela est rendu possible en partant d'interfaces faiblement transmissives puis en les structurant de façon à faire apparaître des modes électromagnétiques de surface qui rendent possible une transmission résonante. En d'autres termes, le rayonnement émis peut devenir partiellement cohérent par filtrage du rayonnement de corps noir existant dans la matière. 
Toutefois, cette remarque n'est pas suffisante pour comprendre l'origine du rayonnement thermique. En effet, le rayonnement émis dépend de la température qui est une grandeur thermodynamique.

Il y a donc d'un côté, le phénomène d'émission qui est de nature thermodynamique ou statistique, de l'autre un phénomène d'absorption par un corps éclairé par une onde cohérente. La loi de Kirchhoff établit un pont entre la thermodynamique et l'optique cohérente, rendu nécessaire par la conservation de l'énergie mais sans nous en fournir le mécanisme physique sous-jacent.

\section{L'ÉMISSION THERMIQUE REVISITÉE AVEC \\ L'ÉLECTROMAGNÉTISME \\ STOCHASTIQUE}

La question de l'origine physique du rayonnement thermique a été traitée par H. A. Lorentz dans son livre "The theory of electrons and its applications to the phenomena of Light and Radiant Heat » publié en 1916 [2]. Sa vision est la suivante: les électrons d'un métal ont un mouvement d'agitation thermique aléatoire de sorte que chaque élément de volume du métal se comporte comme une petite antenne. Le champ émis se propage dans le métal jusqu'à l'interface où il est transmis par l'interface. Dans le cas des métaux, seuls les électrons proches de la surface (quelques dizaines de nanomètres) peuvent contribuer au rayonnement émis. Pour calculer la puissance émise, il faut calculer le flux du vecteur de Poynting. Cette approche a beaucoup d'avantages. Elle permet d'entrevoir le lien entre l'absorption (effet Joule dû aux électrons) et l'émission (rayonnement des courants fluctuants dus aux électrons). Elle permet de fournir une image très simple et très concrète de la loi de Kirchhoff (voir encart). En revanche, à l'époque de Lorentz, les fluctuations thermiques de courant sont décrites dans un modèle classique qui ne permet pas de reproduire la loi de Planck.

\section{L'ÉMISSION THERMIQUE REVISITÉE AU-DELÀ DE L'OPTIQUE GÉOMÉTRIQUE}

La compréhension de la loi de Kirchhoff a fait un bond avec la démonstration du théorème de fluctuation- dissipation. Ce résultat de physique statistique établi en 1951 par Callen et Welton [3] énonce que les fluctuations du courant (les sources $\mathrm{du}$ rayonnement) sont proportionnelles à la partie imaginaire de la permittivité du matériau, qui décrit les pertes, et au facteur de Bose-Einstein $1 /\left[\exp \left(h v / k_{B} T\right)-1\right]$. Dès les années 50 , Rytov a utilisé ce résultat [4] pour redémontrer la loi de Kirchhoff dans un cadre d'électromagnétisme classique parachevant le travail initié par

\section{Figure 1.}

Emission thermique directionnelle par un réseau de SiC (voir schéma). Rouge: mesure de l'émission à 11,26 um; Vert: calcul de de 1-R. Figure adaptée de [5] avec permission de Nature Springer.
Lorentz. Il a introduit des courants aléatoires comme sources des équations de Maxwell: on parle d'électrodynamique stochastique.

Ce nouveau cadre permet d'étendre considérablement la portée de la loi de Kirchhoff. Il montre que l'approximation de l'optique géométrique initialement utilisée dans son raisonnement n'est nullement nécessaire. Dès lors, la loi de Kirchhoff peut s'appliquer à des situations pour lesquelles il y a de la diffraction. Ainsi, il a été montré que la loi de Kirchhoff reste valable pour des particules plus petites que la longueur d'onde bien que la notion de réflectivité d'un rayon sur la surface n'ait plus de sens [4]. On montre que le rayonnement s'exprime en fonction de la section efficace d'absorption prolongeant ainsi naturellement la loi de Kirchhoff.

On peut également concevoir des réseaux ou des métasurfaces résonantes présentant des résonances d'absorption très étroites
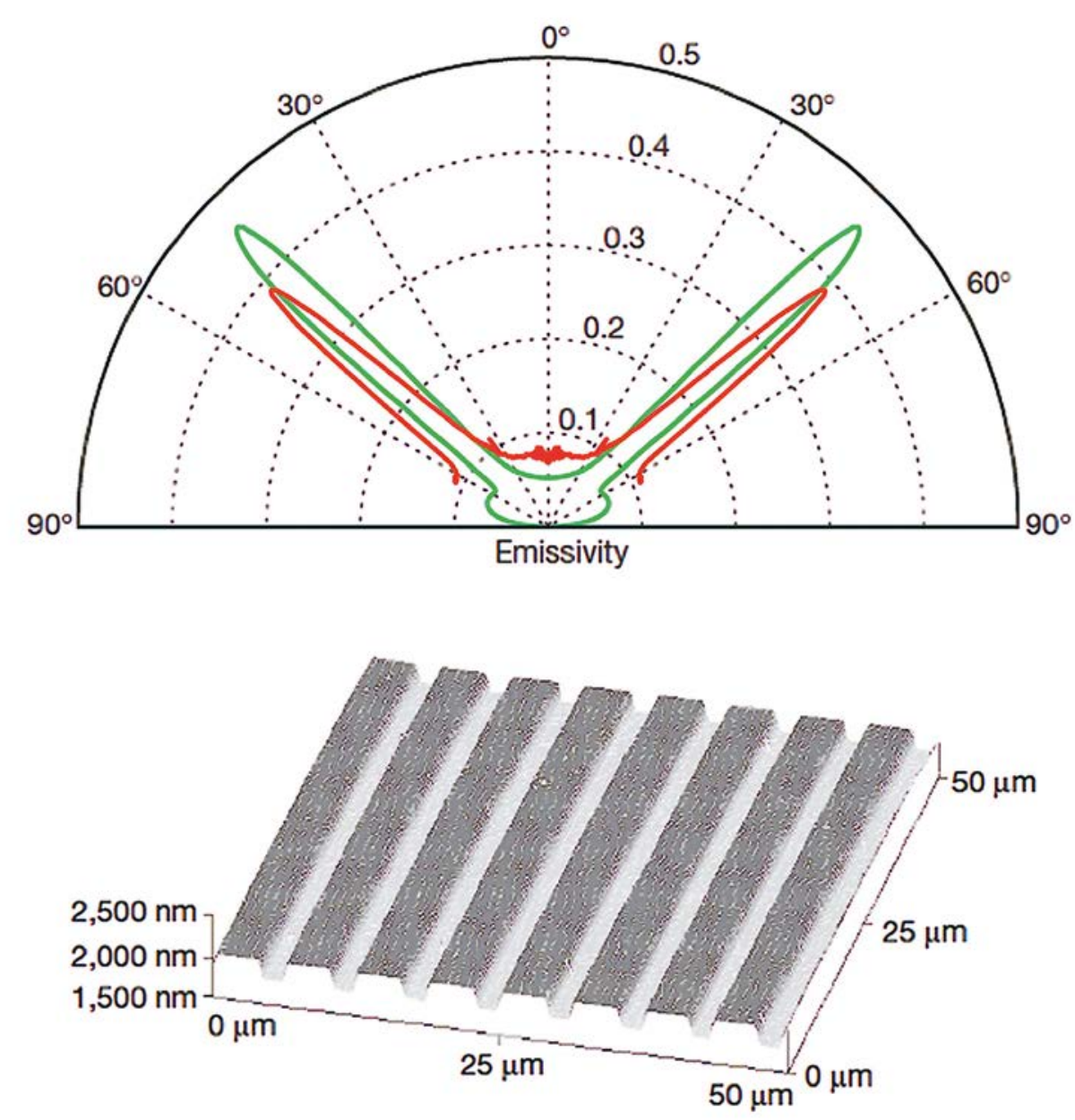


\section{On peut également concevoir des réseaux ou des métasurfaces résonantes présentant des résonances d'absorption très étroites angulairement ou spectralement ce qui conduit donc à de l'émission de rayonnement thermique très directionnelle (spatialement cohérente, ou très monochromatique (temporellement cohérente)}

angulairement ou spectralement ce qui conduit donc à de l'émission de rayonnement thermique très directionnelle (spatialement cohérente, Fig. 1) [5] ou très monochromatique (temporellement cohérente, Fig. 2) [6].

Une autre conséquence de ce formalisme est la capacité à étudier le rayonnement thermique en champ proche qui possède des propriétés très différentes du spectre rayonné en champ lointain. Ceci sort du cadre de la loi de Kirchhoff qui traite de l'émission et de l'absorption d'ondes propagatives. En effet, en champ proche, l'échange d'énergie se fait via des modes non radiatifs (champs décroissant en $1 / r^{3}$ ou ondes évanescentes suivant le point de vue adopté). Dans ce cadre, les flux radiatifs par unité de surface peuvent dépasser de plusieurs ordres de grandeur le flux radiatif $\sigma \mathrm{T}^{4}$ émis par un corps noir.

\section{LA LOI DE KIRCHHOFF}

\section{ET LA LUMINESCENCE}

Bien que historiquement cantonnée à la description du rayonnement thermique, la loi de Kirchhoff est en mesure de décrire les processus d'électroluminescence et de photoluminescence. Une généralisation de cette loi est couramment utilisée pour décrire la lumière émise par des diodes électroluminescentes (LEDs) [7]. Ceci n'est pas surprenant puisque l'émission de lumière par un semiconducteur est une recombinaison radiative électron-trou suivie d'une propagation dans le dispositif. La différence entre le rayonnement thermique, l'électroluminescence et la photoluminescence est la façon de promouvoir un électron de la bande de valence à la bande de conduction tandis que le processus de recombinaison radiative est le même tant que les électrons et les trous sont thermalisés. Il est également possible de généraliser la loi de Kirchhoff à un corps pour lequel différents sous-systèmes sont à des températures différentes [7].

\section{CONCLUSION}

La loi de Kirchhoff a 160 ans et son champ d'application continue de s'étendre. Elle s'applique maintenant à la conception de nouvelles sources de lumière combinant des métamatériaux et des émetteurs quantiques tels que des boîtes quantiques, à la modélisation de l'émission de lumière par des semiconducteurs en cavité ainsi qu'à la compréhension de nouveaux régimes d'émission de lumière tels que les condensats de photons de Bose-Einstein [7].
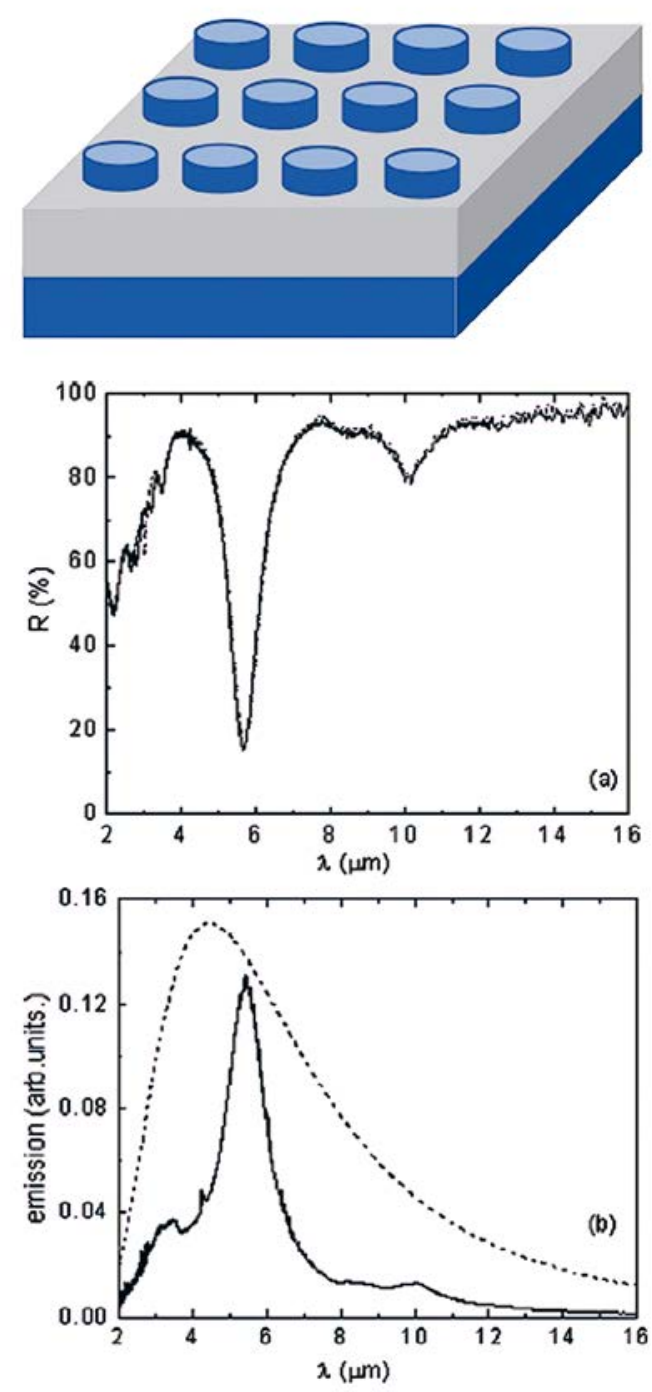

Figure 2.

Métasurface composée d'un miroir d'aluminium recouvert de résonateurs plasmoniques présentant un pic d'absorption, (a) : réflectivité, (b) : spectre d'émission. Figure adaptée de [6] avec permission de AIP Publishing.

\section{RÉFÉRENCES}

[1] G. Kirchhoff, Phil. Mag. S. 4. 20, 1 (1860)

[2] H.A. Lorentz, Theory of Electrons and its applications to the phenomena of light and radiant heat, (B.G. Teubner, Leipzig, 1916)

[3] H.B. Callen, T.A. Welton, Phys. Rev. 83, 34 (1951)

[4] S.M. Rytov, Y.A. Kravtsov, and V.I. Tatarskii, Principles of Statistical Radiophysics vol. 3. (Springer-Verlag, Berlin, 1989)

[5] J.J. Greffet, R. Carminati, K. Joulain et al., Nature (London) 416, 61 (2002)

[6] I. Puscasu, W. Schaich, Appl. Phys. Lett. 92, 233102 (2008)

[7] J.J. Greffet, P. Bouchon, G. Brucoli, F. Marquier, Phys.Rev.X 8, 021008 (2018) 